\title{
COMPORTAMIENTO PRODUCTIVO DE TERNEROS LACTANTES SUPLEMENTADOS CON MAÍZ MÁS TORTA DE ALGODÓN EN EL DEPARTAMENTO DE CÓRDOBA, COLOMBIA
}

\author{
PRODUCTIVE BEHAVIOR OF INFANT'S CALF SUPPLEMENTED WITH CORN \\ MORE COTTON CAKE IN THE DEPARTMENT OF CORDOBA, COLOMBIA
}

\author{
CARDENAS J.E.G, ${ }^{1 *}$ M.Sc, MAZA A.L, ${ }^{2}$ M.Sc, JOSÉ CARDONA Á, ${ }^{3}$ Ph.D. \\ 1*Universidad Federal de Viçosa. Departamento de Zootecnia. Brazil - MG. \\ ${ }^{2}$ Universidad de Córdoba. Grupo de investigación en Producción Animal Tropical, \\ Línea de Nutrición y Alimentación Animal "NUTRA", Montería, Colombia. \\ 3Universidad de Córdoba, Departamento de Ciencias Pecuarias, Semillero de Estudios e Investigaciones en \\ Medicina de Grandes Animales, Grupo MEGA, Montería, Colombia.
}

\section{Key words:}

Supplementation, dual purpose, balanced feed.

\begin{abstract}
To contribute to the search for strategies to improve nutrition and weight gain of calves in livestock systems, weevaluated the growth performance of nursing calves supplemented in double purpose system. 40 calves were used (20 females and 20 males), 1 to 30 days of age with average weights of $26,5 \pm 3,08 \mathrm{~kg}$, which underwent the semi confinement management and supplemented with a feed mixture based on cottonseed meal and ground com in ratios of 1:1, and the administration of hay at will (treated group) and compared with a similar group without supplementation (control group), for 270 days, mainly in the rainy season. Supplemented animals were weaned with a weight adjusted to 270 days of $170 \mathrm{~kg}$., and a weight gain of $531 \mathrm{~g} /$ day, being higher than those found in the control treatment. The weight gain of nursing pups handled in semi confinement was so affected by supplementation means $(p<0.05)$, being supplemented calves daily weight gain $120.3 \%$ higher than the unsupplemented offspring, this may be the contribution of highly digestible nutrients from the supplement and the greatest encouragement in the development of the digestive. The sex of the offspring did notaffect average daily gain of calves within each treatment $(p>0.05)$. The strategy proposed supplement was shown to be technical, biological and economically feasible in young infants dual purpose type.
\end{abstract}

\section{Resumen}

Con el fin de contribuir a la búsqueda de estrategias nutricionales y mejorar la ganancia de peso de temeros en los sistemas pecuarios, se evaluó el comportamiento productivo de temeros lactantes suplementados en un sistema doble propósito. Se utilizaron 40 temeros (20 hembras y 20 machos), de 1 a 30 días de edad y con pesos promedios de $26,5 \pm 3,08 \mathrm{~kg}$, los cuales fueron sometidos al manejo de semiconfinamientoy suplementados con una mezcla alimenticia basada en torta de algodóny maíz molido en proporciones de 1:1, así como la administración de heno a voluntad (grupo tratado) y se compararon œon un grupo similar sin suplementar (grupo œontrol), durante 270 días, principalmente en época de lluvias. Los animales suplementados se destetaron con un peso ajustado a los 270 días de 170 kg., y una ganancia de peso de 531 g/animal/día, siendo mayor a los encontrados en el tratamiento control. La ganancia de peso de las crías lactantes manejadas en semiconfinamiento fue afectada de manerasignificaporlasuplementación $(p<0.05)$, siendoenlas críassuplementadaslagananciadiariade peso $120,3 \%$ superior a las crías no suplementadas, esto puede deberse al aporte de nutrientes de alta digestibilidad por parte del suplemento y al mayor estimulo en el desarrollo del aparato digestivo. El sexo de las crías no afectó la ganancia diaria de peso de los temeros dentro de cada tratamiento $(p>0.05)$. La estrategia suplementar propuesta demostró ser técnica, biológica y económicamente viable en temeros lactantes tipo doble propósito.
Recibido: 09-04-2015;

Aceptado: 01-10-2015.

Correspondencia autor:

yojavier1@hotmail.com 


\section{Introducción}

La situación económica mundial hace que los productores de alimento busquen nuevas estrategias con el fin de alcanzar mejor competitividad, productividad y sostenibilidad, aprovechando la capacidad de los rumiantes para alimentarse de forrajes fibrosos y de subproductos agrícolas e industriales que el hombre no puede consumir o utilizar en forma directa (FRASINELLI et al., 2003).

La actividad ganadera compromete un extenso porcentaje del territorio nacional y el $56 \%$ del PIB pecuario colombiano, sin embargo, su participación en la generación de riqueza, empleo e influencia positiva en los procesos sociales difiere considerablemente en el sistema, manejo, alimentación y cuidado de los hatos bovinos (FEDEGAN, 2006).

Una alternativa importante en la economía colombiana podría ser la actividad ganadera con sistemas doble propósito tropical, definidos como sistemas en los cuales se produce conjuntamente carne y leche, sobre la base de ganado criollo cruzado con cebú y razas lecheras europeas; frecuentemente asociado con la cría de todos los terneros (machos y hembras) mediante amamantamiento directo (CORTÉS et al., 2003).

La dieta láctea es fundamental en la nutrición del ternero especialmente en el primer mes de vida, debido a que sólo el abomaso se encuentra desarrollado para cumplir la función digestiva durante las primeras etapas de vida representando el $70 \%$ del peso del estómago, por lo que la alimentación debe ser mayormente líquida y nutricionalmente concentrada (POND et al., 2007; HILL et al., 2009; BORKERT et al., 2012).

OLIVEIRA et al. (2007) expresan que el desarrollo óptimo de los distintos pre-estómagos o reservorios del ternero se cumplen en tres periodos, entre los cuales se destacan el $1^{\text {er }}$ periodo o pré-rumiante (nacimiento a la $3^{\text {ra }}$ semana de vida), el ternero sólo posee capacidad de digerir leche y depende de la absorción intestinal de glucosa para mantener un valor de glucemia que es semejante al de un no rumiante, el $2^{\circ}$ periodo 0 transición ( $3^{a}$ a la $8^{a}$ semana de vida), en la cual el animal comienza a ingerir pequeñas cantidades de alimento sólido, por lo que estimula gradualmente el desarrollo de los divertículos estomacales, y el 3 er periodo o rumiante (a partir de la $8^{\mathrm{a}}$ semanas de vida), en la cual los divertículos estomacales están bien desarrollados representando el $80 \%$ del estómago lo que permite una digestión fermentativa propia del rumiante adulto.

Las ganaderías del sistema doble propósito ubicadas en la costa Atlántica Colombiana, continúan usando estrategias de manejo inapropiadas que han limitado la producción de leche y carne, a pesar de representar el $38 \%$ de la población bovina nacional, por lo que los terneros lactantes representan una de las categorías más importantes en la producción, debido a que son la fuente de reemplazos de hembras y la fuente de ingresos económicos con la venta de machos destetos (FEDEGAN, 2006).

CAMARGO et al. (2011) manifiestan que en los sistemas tradicionales de crianza, los becerros reciben escaso aporte de leche y ningún tipo de suplementación, por lo tanto, presentan lento crecimiento durante la mejor oportunidad de su vida, lo que afecta negativamente la eficiencia reproductiva y la ganancia de peso.

En busca de mejorar la producción en el sector ganadero doble propósito Colombiano, se hace necesario la investigación de nuevas prácticas y modelos que mejoren los sistemas de producción, por lo que en este estudio se evaluó el comportamiento productivo de terneros lactantes tipo doble propósito suplementados con una mezcla de torta de algodón y maíz molido, así como la administración de heno a voluntad con el fin de establecer su rendimiento en la ganancia diaria de peso, de esta manera generar recomendaciones de manejo nutricional y socializarlo al sector productivo del sistema doble propósito nacional.

\section{Materiales y Métodos}

Tipo de estudio. Se realizó un estudio de tipo experimental, donde se utilizó un diseño completamente aleatorizado, en el que los animales se dividieron en dos grupos para evaluar el efecto de dos tratamientos, uno con suplementación y otro sin suplementación.

Localización. El estudio se realizó en la Hacienda la Ponderosa, municipio de Montería, Córdoba (Colombia), ubicada a 10 m.s.n.m., con una temperatura promedio de $28^{\circ} \mathrm{C}$, topografía plana, $80 \%$ de humedad relativa, precipitación anual de 1100 m.m., su posición geográfica está determinada entre los $8^{\circ} 45^{\prime} 27^{\prime \prime}$ de latitud Norte y 7553'24" de longitud Oeste (PABÓN et al., 2001), a 13 kilómetro de la ciudad.

Aplicación del tratamiento. Cada grupo estaba conformado por 20 animales diez hembras y diez machos cruzados, en proporciones de $3 / 4$ cebú $1 / 4$ taurus, con pesos (26,5 $\pm 3,08 \mathrm{~kg}$ ), edades ( 1 a 30 días) y actitud productiva similares, con el fin de ser comparadas las ganancias de peso promedio y el análisis económico. El estudio fue efectuado durante todo el periodo de amamantamiento, desde el nacimiento hasta el día 270 (9 meses). 
Los animales fueron sometidos al manejo de semiconfinamiento y suplementados con una mezcla de torta de algodón y maíz molido en razón de 1:1 (Tabla 1), así como la administración de heno a voluntad, Angleton (Dichantium aristatum) (Tabla 2) (grupo tratado) y se compararon con un grupo similar donde la única diferencia en el manejo fue el no suministro de suplemento (grupo control), fueron observados durante 270 días principalmente en época de lluvias.

Tabla 1. Composición de materias primas y mezcla suplementaria suministrada a los terneros. Mundo Pecuario (2009).

\begin{tabular}{ccccc}
\hline Nutriente $^{1}$ & Unidad & Maíz & $\begin{array}{c}\text { Torta de } \\
\text { algodón }\end{array}$ & $\begin{array}{c}\text { Mezcla } \\
\text { suplementaria }\end{array}$ \\
\hline MS & $\%$ & 88,00 & 91,00 & 89,50 \\
NDT & $\%$ & 78,00 & 74,00 & 76,00 \\
ED & Mcal/kg & 3,40 & 3,10 & 3,25 \\
EM & Mcal/kg & 3,05 & 2,70 & 2,88 \\
PB & $\%$ & 9,40 & 41,00 & 25,2 \\
Calcio & $\%$ & 0,03 & 0,17 & 0,1 \\
Fosforo & $\%$ & 0,29 & 1,31 & 0,8 \\
Grasa & $\%$ & 3,80 & 1,40 & 2,6 \\
Ceniza & $\%$ & 1,30 & 8,00 & 4,65 \\
Fibra & $\%$ & 2,60 & 13,00 & 7,8 \\
\hline
\end{tabular}

Fuente: Tomado de http://mundo-pecuario.com (2009). ${ }^{1}$ (MS) Materia Seca, (NDT) Nutrientes Digestibles Totales, (ED) Energía Digestible, (EM) Energía Metabolizable, (PB) Proteína Bruta. ${ }^{2}$ Mezcla Alimenticia Calculada

Tabla 2. Composición nutricional de los forrajes utilizados en la alimentación de los terneros. MANTILLA et al. (2010).

\begin{tabular}{ccc}
\hline \multirow{2}{*}{ Nutriente } & \multicolumn{2}{c}{ Materia prima } \\
& Pasto angleton & Heno de angleton \\
\hline \% Materia Seca & 21,38 & 85 \\
$\%$ Proteína Bruta & 13,6 & 11,3 \\
$\%$ FDN & 67,33 & 70,55 \\
$\%$ FDA & 48,1 & 49,6 \\
$\%$ Lignina & 7,1 & 8,7 \\
$\%$ Materia Orgánica & 82,2 & 88 \\
\% Ceniza & 11,8 & 12 \\
\hline
\end{tabular}

Manejo animal. Los animales en estudio fueron sometidos a diferentes tipos de manejo en la medida que aumentaba la edad, por lo que se establecieron cuatro fases claramente definidas: fase 1, desde el primer día de nacidos hasta el primer mes de vida, denominada también fase de acostumbramiento; fase 2, desde el primer mes de vida hasta el cuarto mes, llamado fase de transición; fase 3 , desde el cuarto mes hasta el séptimo mes, o fase de desarrollo; fase 4 comprendida desde el séptimo al noveno mes, momento del destete o fase de finalización (Fig.1). Los terneros del grupo control recibieron el mismo manejo del grupo tratado, a excepción de la suplementación con la mezcla de torta de algodón y maíz molido.
Fase 1. Luego del nacimiento, se suministró calostro durante las primeras 12 horas de vida del neonato y curación de ombligo durante la primera semana de vida por 3 a 5 días. Los terneros fueron alimentados con la mezcla compuesta por maíz y torta de algodón en relación 1:1, alrededor de los 15 días de nacidos, permanecieron todo el tiempo en instalaciones de $132 \mathrm{~m}^{2}$ con piso de tierra y $24 \mathrm{~m}^{2}$ techados para protección de lluvias y sol, además fueron dispuestos comederos con espacios de $30 \mathrm{~cm}$ por animal, con el fin de evitar competencia a la hora de la suplementación, también fueron dispuestos bebederos de 500L., a una altura de $50 \mathrm{~cm}$ para el suministro de agua fresca y limpia, de igual forma, se suministraron $100 \mathrm{~g}$ de la mezcla alimenticia y heno a voluntad. El amamantamiento de los terneros sucedió durante cada mañana al momento del ordeño (3:00 a.m.) y al medio día (12:00 m.) durante 30 minutos, en la cual las vacas fueron llevadas a las instalaciones de confinamiento de los terneros en estudio.

Fase 2. En este periodo los terneros consumían dos porciones de $400 \mathrm{~g}$ cada una, suministrada en horas de la mañana después del ordeño y la otra a las 17:00 horas. El amamantamiento en este periodo fue gradualmente disminuido hasta suspenderlo en las horas de la mañana donde solo eran utilizados para el apoyo y estimulo del ordeño de la vaca. Al medio día, se llevaban las vacas por 30 minutos al lugar de confinamiento de los terneros para el amamantamiento y continuaban con el suministro de heno a voluntad.

Fase 3. En este periodo los terneros solo ingerían leche residual y eran utilizados para el apoyo y estimulo del ordeño de la madre. Después del ordeño se les suministro una porción de $300 \mathrm{~g}$ de la mezcla, para luego ser llevados a potreros de 0.5 hectáreas con pasto Angleton (Dichantium aristatum) (Tabla 2), teniendo en cuenta que se encontraban en buena condición de cantidad y calidad nutricional, así mismo fueron rotados en sistema de franjas con periodos de descanso de 21 días y 2 de ocupación, después de este proceso (al medio día), eran llevados a los potreros donde se encontraban las madres para ser amamantados por 30 minutos, para después ser llevados al potrero de pastoreo. A las 5:00 de la tarde se les suministró la segunda porción de $300 \mathrm{~g}$ en el módulo de confinamiento y el suministro de heno a voluntad.

Fase 4. En esta fase los terneros fueron usados sólo para el estímulo al momento del ordeño, luego eran llevados al módulo de semiconfinamiento donde se le suministraba la primera porción del día (250 g de la mezcla alimenticia), después eran llevados a los potreros de pastoreo donde permanecieron todo el día hasta las 17:00 horas y posterior a esto eran llevados nuevamente al módulo de confinamiento donde se les suministraba la segunda porción de $250 \mathrm{~g}$ de la mezcla, continuando siempre confinados y consumiendo heno a voluntad. 


\section{Fict}

Cardenas, et al.

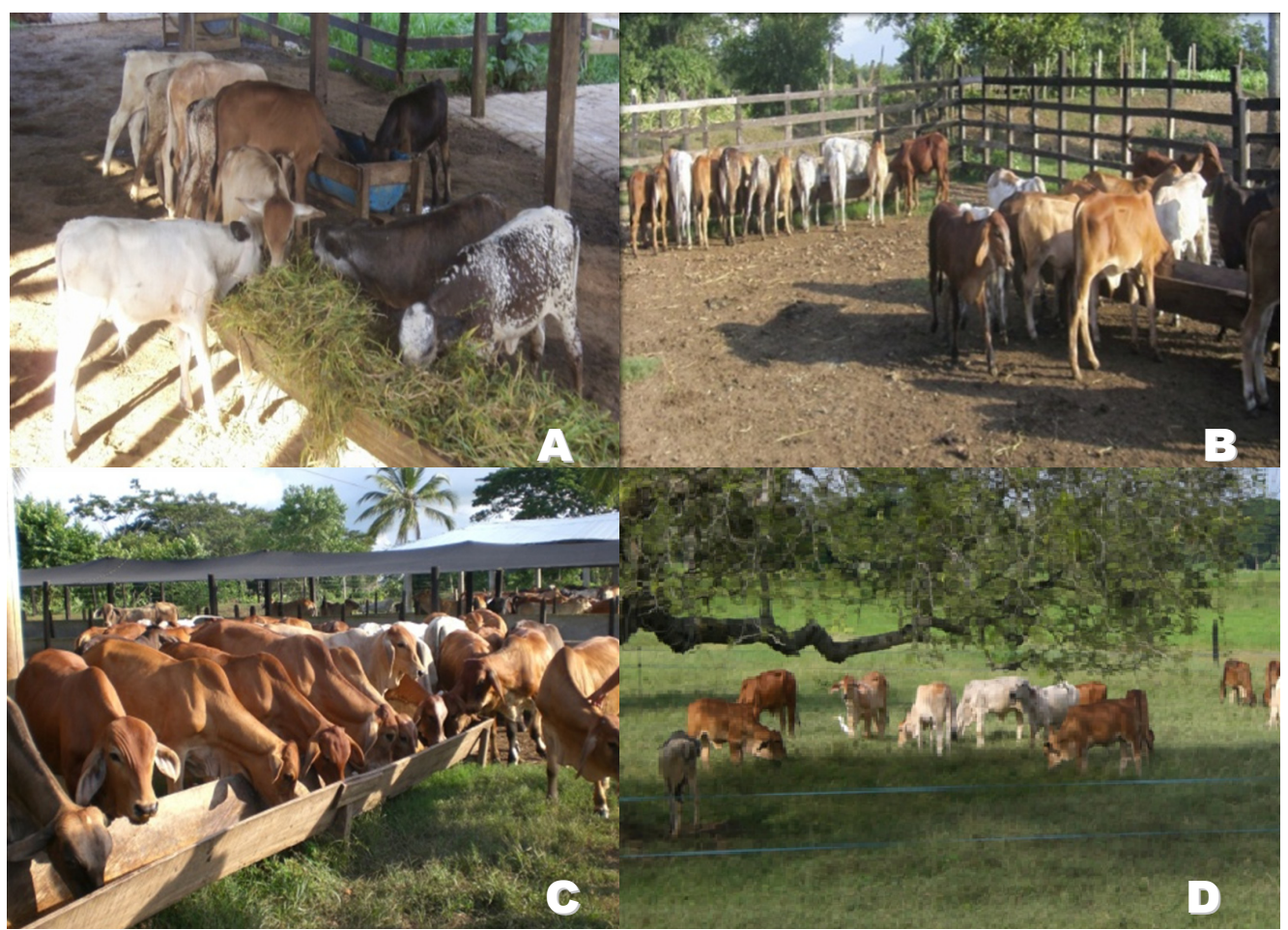

Figura 1. Imágenes de la investigación. A: Fase 1, B: Fase 2, C: Fase 3, D: Fase 4.

Determinación del peso. La ganancia de peso, fue evaluada con un seguimiento de pesajes a los animales cada 15 días, en el cual los animales eran pesados individualmente en una báscula electrónica, en total cada animal fue pesado 18 veces. Se estableció el peso ajustado a los 270 días según la edad de cada animal, ya que según Montes et al. (2009), este cálculo elimina la influencia del ambiente, ocasionadas por edades de nacimiento diferentes, para este cálculo fue utilizada la siguiente formula:

\section{$\mathrm{PA} 270=[(\mathrm{PD}-\mathrm{PN}) / \mathrm{E}]^{\star} 270+\mathrm{PN}$}

PA270: Peso al destete ajustado a 270 días. PD: Peso al destete. PN: Peso al nacer. E: Edad al destete en días.

Análisis estadístico. Con la información de campo obtenida se realizó la prueba t de Student para determinar la diferencia estadística $(P \leq 0,05)$ entre las medias de los tratamientos utilizados con relación a la ganancia de peso.

Análisis económico. Para el análisis económico, se usó la técnica del presupuesto parcial propuesta por MURCIA (1985). Se tuvieron en cuenta los aumentos adicionales en el costo total por concepto de la suplementación basado en el precio de las materias primas que conforman la mezcla alimenticia y el consumo del suplemento por animal en cada una de las fases, considerando los efectos simples de los tratamientos en donde se compararon las medias obtenidas para cada variable por tratamiento. Se determinó la relación beneficio costo, la utilidad neta y la rentabilidad. El precio por $\mathrm{kg}$ de peso fue considerado de acuerdo al precio de venta de animales en subastas de la zona.

\section{Resultados y Discusión}

La ganancia de peso promedio en los animales suplementados fue de $531 \mathrm{~g} / \mathrm{d}$ ía, siendo mayor a la ganancia de peso del grupo control que fue de $241 \mathrm{~g} /$ día, por lo que se obtuvo una ganancia de peso adicional de $290 \mathrm{~g} /$ día $(\mathrm{P} \leq 0,05)$. De igual forma el peso promedio al destete fue calculado a una edad ajustada de 270 días, en el grupo tratado fue de $170 \mathrm{~kg}$, siendo mayor al peso promedio al destete del grupo control que fue de 91,5 $\mathrm{kg}$, por lo que se tuvo una ganancia adicional de 78,5 $\mathrm{kg}(\mathrm{P} \leq 0,05)$. La ganancia de peso de las crías lactantes manejadas en semiconfinamiento fue afectada de manera significativa $(P \leq 0,05)$ por la suplementación con una mezcla de torta de algodón y maíz molido más el suministro de heno a voluntad, pudiendo atribuirse esto al aporte de nutrientes de alta digestibilidad por parte del suplemento y al mayor estimulo en el desarrollo del aparato digestivo. El sexo de las crías no afectó la ganancia diaria de peso de los terneros dentro de cada tratamiento $(P \geq 0,05)$. Es importante indicar que 
no se presentaron enfermedades, disminuyendo la morbilidad y mortalidad en los animales suplementados, concordando con lo informado por GONZÁLEZSTAGNARO et al. (2006), quienes manifiestan que uno de los beneficios de la suplementación es la disminución de enfermedades predestete.

Los resultados obtenidos en este estudio con respecto a la ganancia de peso promedio de $531 \mathrm{~g} /$ día en los terneros suplementados, se encuentra dentro del rango de ganancias de peso significativas según PAULINO (1998), quien expresa que el aporte de nutrientes vía suplemento durante las fases de crecimiento afecta el desempeño de los animales, obteniéndose ganancias moderadas de 200 a 300 g/día que pueden alcanzar ganancias significativas de 500 a $600 \mathrm{~g} /$ día en animales suplementados. GARZÓN (2007) explica que la utilización de suplementación fibroso-proteica y energética, además de contribuir con los requerimientos nutricionales de los terneros, inducen a mayor desarrollo ruminal y un mejor establecimiento de la microbiota, acciones que facilitan una digestión superior de los alimentos fibrosos, sin embargo aclara que la edad en que se produce el cambio de la digestión monogástrica a la de rumiante depende estrechamente de la dieta utilizada, por lo que se podría explicar la facilidad de adaptación al alimento a base de forraje en el presente estudio. Así mismo CANELONES y CASTEJON (2006) explican que a partir de la mayor síntesis de compuestos nitrogenados, se aumenta la producción activa de vitamina B y de proteína. Autores como CAÑAS et al. (2008), manifiestan que es esperado que los animales suplementados con alimentos balanceados comerciales, presenten mayores ganancias de peso, ya que las materias primas tienen mejores digestibilidades que los subproductos agroindustriales.

Ganancias diarias de peso inferiores a la de este estudio han sido reportadas por autores como OVIEDO et al. (2011), quienes obtuvieron ganancias de 484 g/día en terneras lactantes manejadas bajo el sistema doble propósito, suplementadas con semilla de algodón y melaza, en el departamento de Córdoba, Colombia; SIDIBÉ-ANAGO et al. (2008), reportaron ganancias muy inferiores $(149 \mathrm{~g})$ en terneros doble propósito Cebú, alimentados con heno, torta de algodón y melaza a voluntad, en Burkina Faso, África; GONZÁLEZSTAGNARO et al. (2006), obtuvieron ganancias de $452 \mathrm{~g} /$ día en terneras lactantes de doble propósito suplementas con alimento balanceado comercial en el Estado de Zulia-Venezuela; MARTíNEZ et al. (2001), reportaron ganancias de $500 \mathrm{~g} /$ día suplementando terneros doble propósito con un alimento balanceado comercial; BRAVO y BRACHO (2000), reportaron ganancias de $378 \mathrm{~g} /$ día en terneros criollos Limoneros en pastoreo y suplementados con un alimento balanceado de $18 \%$ de proteína cruda, en el estado del Zulia-Venezuela.
Ganancias diarias de peso superiores a las de este estudio han sido reportadas en terneros doble propósito, tal es el caso de PRIETO et al. (2010), quienes reportaron ganancias superiores con $870 \mathrm{~g} /$ día en terneros lactantes doble propósito suplementados con un alimento balanceado comercial el departamento de Sucre, Colombia; JABBAR et al. (2006), reportaron ganancias de $730 \mathrm{~g} / \mathrm{día}$ en terneros mestizos suplementados con torta de algodón a voluntad en Sahiwal, Pakistan. Así mismo, REZA et al. (2003), estudiaron el efecto de la suplementación de terneros lactante bajo un sistemas de doble utilidad con semilla de algodón, obteniendo ganancias de 583 g/día; KaBEYA et al. (2002), reportaron ganancias de $840 \mathrm{~g} /$ día suplementando animales a con un alimento a base de maíz y torta de soya.

Los resultados favorables en el grupo tratado que recibió suplementación, con respecto al grupo control, podría explicarse en la adaptación fisiológica que los animales desarrollaron a lo largo del estudio. WATTIAUX (1997) y GOES et al. (2005), informan que los bovinos jóvenes cuando son alimentados con leche, se cierra la escotadura esofágica haciendo que sobrepase el retículo-rumen y fluya directamente hacia el abomaso.

Sin embargo, cuando se ingieren alimentos sólidos, la escotadura esofágica gradualmente cesa su función y una población bacteriana se establece en el rumen, y comienza el desarrollo de la pared ruminal, ya que estimula el desarrollo de los estratos epiteliales y la queratinización, ocurriendo cuatro semanas después de la introducción de alimento sólido en la dieta, también aumenta el grosor de la musculatura del reticulo-rúmen, cuando el rumiante recién nacido tiene acceso al pasto, este comienza a pastar en la primera y segunda semana de vida, iniciando el desarrollo de los compartimientos estomacales retículo-rumen (COPPO, 2007).

Dietas carentes de material fibroso pueden originar formación anormal de papilas ruminales y de la queratinización en la mucosa del rumen, resultando en problemas en la absorción de nutrientes, debido a que la leche como único alimento tiene poco efecto sobre el desenvolvimiento del epitelio, siendo la administración de alimentos sólidos, como concentrado, forraje, o los dos juntos, una buena estrategia para mejorar el desenvolvimiento del retículo-rumen (OLIVEIRA et al. 2007).

En el análisis económico basado en la técnica de presupuesto parcial, se determinó el costo del sistema de suplementación en $€ 47.37$ euros/animal (Tabla 3) durante la fase de lactancia, así mismo se obtuvieron ingresos por venta de $€ 123,24$ euros por cada animal del grupo testigo y $€ 228,97$ euros por venta de cada animal del grupo experimental, resultando en un 
incremento del ingreso por venta de $€ 105,73$ euros por animal del grupo suplementado con respecto al no suplementado, obteniéndose así un beneficio económico neto por animal de $€ 58,36$ (Tabla 4) a favor del grupo experimental y una tasa de retorno marginal de 2.23, es decir que por cada euro invertido en el sistema se recuperan $€ 1,23$ euros.

Tabla 3. Costo general de la suplementación en todo el periodo experimental/Animal.

\begin{tabular}{ccc}
\hline Fase & $\begin{array}{c}\text { Cantidad y precio por kg de } \\
\text { suplemento }\end{array}$ & Valor $€$ \\
\hline 1 & $0,1 \mathrm{~kg} / 30$ días/€0,29 Costo $\mathrm{kg}$. & 0,87 \\
2 & $0,8 \mathrm{~kg} / 90$ días/€0,29 Costo $\mathrm{kg}$. & 20,89 \\
3 & $0,6 \mathrm{~kg} / 90$ días/€0,29 Costo $\mathrm{kg}$. & 15,67 \\
4 & $0,5 \mathrm{~kg} / 60$ días/€0,33 Costo $\mathrm{kg}$. & 9,95 \\
\hline Total & & $\mathbf{4 7 , 3 7}$ \\
\hline
\end{tabular}

Tabla 4. Análisis económico mediante la técnica del presupuesto parcial/Animal.

\begin{tabular}{ccc}
\hline Ítems & Grupo testigo & Grupo experimental \\
\hline Costo variable $(€)$ & $123,24^{*}$ & 47,37 \\
Ingreso por venta $(€)$ & $228,97^{\star}$ \\
Incremento en el ingreso $(€)$ & 105,73 \\
Relación beneficio - costo & 2,23 \\
Beneficio Económico Neto $(€)$ & $\mathbf{5 8 , 3 6}$ \\
\hline${ }^{*}$ Costo por kg de peso de los animales vendidos en subasta comercial, $€ 1,35$.
\end{tabular}

Es importante mencionar que tasas de retorno marginal inferiores a las del presente estudio, fueron reportadas por PRIETO et al. (2010), quienes al suplementar terneros lactantes con alimento balanceado comercial en sistemas doble propósito, obtuvieron un ingreso marginal de 1,1; así mismo OVIEDO et al. (2011) informaron ingresos marginales de 1,73 al suplementar terneras lactantes doble propósito con semilla de algodón y melaza durante la época seca en el valle medio del Sinú.

\section{Conclusiones}

De esta investigación se concluye que los animales suplementados presentaron ganancias diarias de peso promedio mayor con respecto a los del grupo control, así como mejor peso promedio al destete. De igual forma la mejora en la condición corporal, disminuye considerablemente la morbimortalidad al establecer una estrategia de suplementación con ventajas para los productos regionales o subproductos agroindustriales, que además es económicamente viable como lo sugiere este estudio.

Agradecimientos. Los autores expresan sus más sinceros agradecimientos al propietario de la Finca La Ponderosa, en la ciudad de Montería, Colombia, por su valiosa colaboración para el desarrollo de esta investigación. Financiación: Este estudio fue financiado por la Facultad de Medicina Veterinaria y Zootecnia de la Universidad de Córdoba, Montería, Colombia. Conflictos de intereses: El manuscrito fue preparado y revisado con la participación de todos los autores, quienes declaramos que no existe ningún conflicto de intereses que ponga en riesgo la validez de los resultados presentados.

\section{Referencias}

BORKERT, J.; NORO, M.; PULIDO, R.; WITTWER, F. 2012. Respuesta metabólica de terneros de lechería alimentados con sustituto lácteo nacional o importados. Revista Científica, FCV-LUZ. 22(2):171-176.

BRAVO, J.; BRACHO, I. 2000. Respuesta de becerros criollo limonero sometidos a diferentes niveles de suplementación. Revista Facultad de Agronomía. LUZ. 17: 282-287,

CAMARGO, M.; PÁRRAGA, C.; MEJÍA, E.; ESCOBAR, A.; COLMENÁREZ, M. 2011. Subsistemas de crianza de becerros y su relación con el desarrollo de fincas doble propósito en el estado portuguesa. Rev. Unellez Cienc. Tecnol. 29: 39-46.

CANELONES, C; CASTEJON, M. 2006. Harinas de planta entera de frijol (Vigna unguiculata) y de mazorca de maíz (Zea mays) como suplemento para becerros antes del destete. Zoot. Tropical. 24 (3): 361-378.

CAÑAS, J.; RAMÍREZ, J.; ARBOLEDA, O.; OCHOA, J.; VERGARA, O.; CERÓN-MUÑOZ, M. 2008. Estimación de parámetros genéticos para peso al destete en ganado Blanco Orejinegro (BON) en el noroccidente colombiano. Rev. MVZ Córdoba. 13: 1138-1145.

COPPO, J. 2007. ¿El destete precoz produce estrés en los terneros cruza cebú?. Rev. Electrón. Vet. VIII (2):1-28. Disponible en: http://www.veterinaria.org/revistas/recvet/n020207/020719.pdf Accesado el 29/05/12. 
CORTÉS, H.; AGUILAR, C.; VERA, R. 2003. Sistemas bovinos doble propósito en el trópico bajo de Colombia. Modelo de simulación. Archivos Zootecnia. 52: 25-34.

FEDEGAN. 2006. Plan estratégico de la ganadería colombiana 2019. Primera Ed. Bogotá, Colombia. Ed. Sanmartín Obregón \& Cía. p.26-28. Disponible en: http://portal.fedegan.org.co/Documentos/pega 2019.pdf Accesado el $30 / 05 / 2012$.

FRASINELLI, C.; VENECIANO, J.; BELGRANO-RAWSON, A.; FRIGERIO, K. 2003. Sistemas extensivos de producción bovina: productividad y rentabilidad. En: Aguilera, M.; Panigatti, J. (Ed.) Con las metas claras. La EEA San Luis: 40 años a favor del desarrollo sustentable. Argentina. Ed. INTA. cap. 8, p.141-157.

GARZÓN, B. 2007. Sustitutos lecheros en la alimentación de terneros. Rev. Electrón. Vet. VIII (5): 1-39. Disponible en: http://www.veterinaria.org/revistas/redvet/n050507/050701.pdf Accesado el 27/05/12.

GOES, R.; MANCIO, A.; LANA, R.; ALVES, D.; LEÃO, M.; SILVA, A. 2005. Recria de novilhos mestiços em pastagem de Brachiaria brizantha, com diferentes níveis de suplementação, na região Amazônica. Desempenho Animal. Rev. Bras. Zootec. 34 (5): 1740-1750.

GONZÁLEZ-STAGNARO, C.; RODRÍGUEZ-URBINA, M.; GOICOCHEA-LLAQUE, J.; MADRID-BURY, N.; GONZÁLEZ-VILLALOBOS, D. 2006. Crecimiento pre-destete en hembras bovinas doble propósito. Rev. Cient. FCV-LUZ. 16 (3):.288-296.

HILL, T.; BATEMAN, H.; ALDRICH, J.; SCHLOTTERBECK, R. 2009. Effects of fat concentration of a high protein milk replacer on calf performance. J. Dairy Sci. 92: 5147-5153.

JABBAR, M.; ANJUM, M.; REHMAN, S; SHAHZAD, W. 2006. Comparative efficiency of sunflower meal and cottonseed cakes in the feed of crossbred calves for meat production. Pakistan Vet. J. 26: 126-128.

KABEYA, K.; PAULINO, M.; DETMANN, E.; VALADARES-FILHO, S.; CECON, P.; QUEIROZ, D.; GOMES-JÚNIOR, P.; PEREIRA, O. 2002. Suplementação de novilhos mestiços em pastejo na época de transição água-seca: Desempenho produtivo, características físicas de carcaça, consumo e parâmetros ruminais. Ver. Bras. Zootec. 31 (1): $213-222$.

MARTÍNEZ, M.; BRAVO, J.; BETANCOURT, M.; MORÁN, V. 2001. Efecto de la suplementación sobre el crecimiento de becerros mestizos en la época seca. Zootec. Trop. 19 (1): 31-42.

MANTILLA, C.; OVIEDO, L.; BETANCUR, C. 2010. Efecto de la época de corte sobre la composición química y degradabilidad ruminal del pasto Dichanthium aristatum (Angleton). Zootec. Trop. 28 (2): 275-281.

MONTES, D.; VERGARA, O.; PRIETO, E.; BARRAGÁN, W. 2009. Estimación de la repetibilidad y factores que afectan el peso al nacer y al destete en ganado bovino cebú brahmán. Rev. Col. Cienc. An. 1(1): 19-36.

MUNDO PECUÁRIO. 2009. Matérias primas para la elaboración de raciones para rumiantes. Disponible en: http:// mundo-pecuario.com/tema61/nutrientes_para_rumiantes.html Accesado el 06/06/12.

MURCIA, H. 1985. Administración de empresas asociativas de producción agropecuaria. IICA. Costa Rica. 242p.

OLIVEIRA, J.; ZANINI, M.; SANTOS, E. 2007. Fisiologia, manejo e alimentação de bezerros de corte. Arq. Ciênc. Vet. Zootec. 10(1): 39-48.

OVIEDO, C.; PASTRANA, A. MAZA, L.; SALGADO, R.; VERGARA, R. 2011. Suplementación de terneras lactantes doble propósito en la época seca en el valle medio del Sinú, Colombia. Rev. U.D.C.AAct. Div. Cient. 14 (1): $57-62$.

PABÓN, J.; ESLAVA, J.; GÓMEZ, R. 2001. Generalidades de la distribución espacial y temporal de la temperatura del aire y de la precipitación en Colombia. Meteorol. Col. 4: 47-59.

PAULINO, M. 1998. Suplementos múltiplos para recria e engorda de bovinos em pastagens. Anais do congresso nacional dos estudantes de zootecnia, Viçosa, Brasil. 173-188. 
POND, W.; CHURCH, D.; POND, K. 2007. Fundamentos de nutrición y alimentación de animales. $2^{\text {da }}$ ed. México. Ed. Limusa S.A. p.349.

PRIETO, E.; MONTES, D.; LARA, L.; RÍOS, R. 2010. Suplementación con balanceado comercial en crías vacunas lactantes bajo sistema doble propósito. Rev. MVZ Córdoba. 15 (3): 2194-2203.

REZA, S.; CUADRADO, H.; MARTíNEZ, J; ALVARADO, L.; ABUABARA, Y.; TORREGROSA, L. 2003. Análisis Técnico - Económico de un manejo de alimentación para bovinos lactantes en el sistema doble propósito en el valle del Sinú. Carta Fedegan. 78: 82-90.

SIDIBÉ-ANAGO, A.; OUEDRAOGO, G.; LEDIN, I. 2008. Effect of suckling period on calf growth and milk yield of zebu cows. Trop. An. Health and Prod. 40 (7): 491-499.

WATTIAUX, M. 1997. Crianza de terneras y novillas. Instituto Babcock para Investigación y Desarrollo Internacional para la Industria Lechera. USA. 134p. 